\author{
David Jarjoura, ${ }^{1}$ Ph.D.; James Jamison, ${ }^{1}$ Ph.D.; and \\ Stavroula Androulakakis, ${ }^{1}$ M.S.
}

\title{
Likelihood Ratios for Deoxyribonucleic Acid (DNA) Typing in Criminal Cases
}

\author{
REFERENCE: Jarjoura, D., Jamison, J., and Androulakakis, S., "Likelihood Ratios for \\ Deoxyribonucleic Acid (DNA) Typing in Criminal Cases,' Journal of Forensic Sciences, \\ JFSCA, Vol. 39, No. 1, January 1994, pp. 64-73.
}

\begin{abstract}
The likelihood ratio approach for DNA typing in criminal cases is described. It is shown how this approach uses both the sizes and pattern of discrepancies between the crime scene profile of fragment lengths and the suspect profile for quantifying the strength of the evidence. In contrast to the current match-binning approach, it avoids an initial decision about whether the two profiles match. Likelihood ratios for pairs of profiles that meet the published statistical criteria for a match show a wide range of values including some that indicate the evidence is strongly against identity.
\end{abstract}

KEYWORDS: pathology and biology, DNA, criminalistics, likelihood ratios

A likelihood ratio approach to the statistical presentation of DNA fragment length evidence differs from the approach referred to as match-binning in the manner in which it quantifies the comparison between the profile of DNA fragment lengths obtained from a sample at the crime scene and that obtained from a suspect. Unique to match-binning is an initial decision about whether the profiles match. Under match-binning a match is declared when the two profiles reveal no significant discrepancies. A chance-matching probability is then determined from the population frequency of fragment lengths that fall in the matching interval or bin. Although expert judgement is required for declaring a match, statistical-matching criteria have been published that require that each pair of fragment lengths in the two profiles differ by less than four standard errors of measurement [1] or $2.5 \%$ of the fragment lengths [2]. A more stringent three standard error criteria has also been reported [3]. Recent debates over the statistical validity of matchbinning have focused upon the estimation of the probability of matching profiles from two different people $[1,4,5]$. A committee of the National Academy of Sciences reported on this and other issues and proposed a conservative method for calculating these chancematching probabilities [6]. A more important statistical issue is whether it is sufficient to provide conservative, but convincing, chance-matching probabilities after a match has been declared; or better to use the sizes and pattern of discrepancies between two profiles in characterizing the strength of the evidence.

Received for publication 23 Nov. 1992; revised manuscript received 8 Feb. and 17 May 1993; accepted for publication 14 June 1993.

${ }^{1}$ Associate Professor of Biostatistics, Community Health Sciences; Research Instructor, Microbiology/Immunology; Research Associate, Community Health Sciences; respectively, Northeastern Ohio Universities College of Medicine, Rootstown, $\mathrm{OH}$. 
The likelihood ratio approach as an alternative to match-binning does use the sizes and pattern of discrepancies to quantify the strength of the evidence. A likelihood ratio indicates the evidence is stronger when a pair of profiles match closely than when a pair barely meet statistical-matching criteria. Because of the reported positive correlation of fragment length measurement errors of the alleles, a likelihood ratio also indicates the evidence is stronger when a pair of profiles exhibit differences of the same sign for the alleles than when a pair exhibit differences of opposite sign. Under match-binning, the sizes and pattern of discrepancies is considered by experts in the match decision, but the closeness of the match is not quantified.

For DNA typing in criminal cases, the numerator of the likelihood ratio is the likelihood of a model that assumes the suspect and crime sample fragment length profiles are identical, and the denominator is the likelihood of a model that does not assume identical profiles. The models logically follow from consideration of the distribution of errors in measuring fragment lengths and from the distribution of fragment lengths in target populations. Likelihood ratio approaches for DNA typing in criminal cases have been proposed by others [7-12] and have long been suggested for other forensic evidence [13]. For DNA typing in paternity cases, likelihood ratios have been referred to as the paternity index $[14,15]$. We provide a relatively simple likelihood ratio equation that considers the reported complexities of the fragment length measurement error distribution in quantifying the strength of the evidence. Application of this equation reveals the importance of the sizes and pattern of discrepancies between profiles. We also show that when two profiles are not too close, but still meet a four-standard-error statistical-matching criteria, the likelihood ratio approach indicates that the evidence is strongly against identity. With the use of the likelihood ratio approach, it becomes more apparent that accurate estimation of the fragment length measurement error distribution $[2,3,8,16]$ and expertise in the various conditions that keep it under control [17-19] are at least as critical as the determination of chance-matching probabilities $[1,4-6]$.

\section{A Likelihood Ratio for DNA Typing}

A simple measurement error model posits a true fragment length $T$ as the expected value of the measured values $X$, such that $X=T+E$, where $E$ represents measurement error. A coefficient of variation of just $0.6 \%$ of the fragment length has been frequently reported for Lifecodes methodology and was based on comparisons of duplicate, uncontaninated samples $[1,3,7]$, but the particular value is not important to our presentation. Because of the numerous conditions that contribute to the small variance of $E$ and from the results of empirical studies $[8,16]$, it seems reasonable to assume $E$ to be distributed normally with mean zero and variance $c^{2} T^{2}$, where $c$ is the coefficient of variation. For the two fragment length measurements (one for each allele) obtained from each probe (locus), we use $X$ to denote the observed shorter allele and $Y$ to denote the longer allele; where $Y=U+F, U$ is the true fragment length of the longer allele, and $F$ is the measurement error variable assumed normal with mean zero and variance $c^{2} U^{2}$. Because of indications of high positive correlations between errors $E$ and $F$ when $T$ and $U$ are close, we adopted a model that allows them to be dependent. As we show below, such correlations are a critical component in the modeling and the resulting likelihood ratios. Error correlations ranging from 0.25 to 0.9 have been reported $[7,8,12]$, and functions for the error correlations that depend on the difference between the two alleles have been suggested $[9,12]$. The correlations, as one would expect, approach one as the difference between alleles approaches zero.

The density function that can be estimated by identifying a sample of individuals from some target population, sorting within each pair of alleles a short and long measured 
length, and smoothing bivariate frequencies can be written in terms of the measurement model,

$$
h(x, y)=\iint g(t, u) \phi(x, y \mid t, u) d t d u
$$

where $g(t, u)$ is the bivariate density of the true fragment lengths $(t, u)$, and $\phi(x, y \mid t, u)$ is the conditional (error) density of the observed fragment lengths $(x, y)$ for a given $\mathrm{t}$ and $u$. The symbol $\phi$ refers to the bivariate normal error assumption $[(X, Y \mid t, u)$ is normal with means $t$ and $u$; variances $c^{2} t^{2}$ and $c^{2} u^{2}$, and covariance $\rho c^{2} u t$, where $\rho$ is the correlation of the error variables $E$ and $F$ and is a function of the difference between $t$ and $u$ ].

Data on three probes (D2S44, D17S79, and D14S13) were made available to us from Lifecodes Corporation. We found that across the three probes and across three racial groups the correlations between $X$ and $Y$ for each probe ranged between 0.34 and 0.52 . The intraclass correlation which ignore order were lower than these $(0.01$ to 0.18$)$. Because of the size of these correlations, we used a bivariate density for the $T$ and $U$ alleles that allowed them to be dependent. Therefore, Hardy-Weinberg Equilibrium, which has been a source of criticism in many previous approaches, is not assumed in our model [20]. However, there is empirical evidence that the Hardy-Weinberg assumption yields accurate statistics [1,9].

When the suspect is the perpetrator the DNA fragments from the suspect and crime samples are expected to be identical lengths. For a single probe, therefore, the observed shorter fragment lengths from the two samples are written in terms of a common $T$, $X_{s}=T+E_{s}$ and $X_{p}=T+E_{p}$, where $s$ refers to the suspect sample and $p$ the crime sample. The error variables $E_{s}$ and $E_{p}$ are assumed independent, because they are from different samples and are typically measured in separate lanes. Similarly, for the pair of longer fragment lengths with common true length, we write, $Y_{s}=U+F_{s}$, and $Y_{p}=U$ $+F_{p}$, where again $F_{s}$ and $F_{p}$ are assumed independent. The likelihood that a common $T$ and $U$ gave rise to the four observations $x_{s}, x_{p}, y_{s}$, and $y_{p}$ is simply the density of these observations given a common $T$ and $U$.

When the suspect is not the perpetrator, the true fragment lengths from the two samples are not expected to be identical, that is, the observed fragment lengths are not written with a common $T$ and $U, X_{s}=T_{s}+E_{s}, X_{p}=T_{p}+E_{p}, Y_{s}=U_{s}+F_{s}$, and $Y_{p}=U_{p}+F_{p}$. The likelihood of the second measurement model as the explanation for the four observations is the density of their values without the assumption that $T_{s}=T_{p}$ and $U_{s}=U_{p}$.

The likelihood ratio (LR) is then written as

$$
\mathrm{LR}=\frac{f\left(x_{s}, x_{p}, y_{s}, y_{p} \mid s=p\right)}{f\left(x_{s}, x_{p}, y_{s}, y_{p} \mid s \neq p\right)},
$$

where $f$ denotes the two density functions, $s=p$ denotes identical true fragment lengths underlying the suspect and crime samples, and $s \neq p$ denotes no such assumption.

The greater the value of the LR (above 1), the more strongly the evidence favors the identity (numerator) model. Conversely, the lower the value (below 1), the more strongly the evidence favors the non-identity (denominator) model. Some labeling scheme seems the easiest method for conveying the meaning of an LR value to lay persons. Evett et al. [10] provide a scheme in which an LR over 1000 is labeled: "the evidence very strongly supports" identity. A labeling scheme in combination with empirical studies of its inaccuracies could be the foundation of the statistical presentation in a courtroom. For example, if the LR were greater than 1000 , the above label could be followed by a 
discussion of the proportion of LRs over 1000 that have been obtained from comparing the profiles of two different people in a large data base. Presumably, such large values for the LR would almost never arise from two different people (excluding monozygotic twins) even in the largest data bases. For example, using just two probes to compare profiles of different persons, Evett et al. [12] found that only one in one-half million comparisons produced an LR greater than 1000. They also found that, with just two probes, $98 \%$ of LR values exceeded 10000 when based on duplicate samples from the same person.

\section{A Likelihood for the Identity Model in Closed Form}

The density of $x_{s}, x_{p}, y_{s}$, and $y_{p}$ for one locus given identical true fragment lengths (common $T, U$ ) for the two samples is,

$$
f\left(x_{s}, x_{p}, y_{s}, y_{p} \mid s=p\right)=\iint g(t, u) \phi\left(x_{s}, y_{s} \mid t, u\right) \phi\left(x_{p}, y_{p} \mid t, u\right) d t d u
$$

where again $g(t, u)$ is the population bivariate density of the true short and long fragment lengths. When measurement error variance is relatively small as it is here, a Taylor series approximation has been found to yield accurate results for the integration in Eq 3 [21,22]. The main part of the approximation involves the bivariate normal density of the errors in the observations and the bivariate density $h(x, y)$ of short and long fragment lengths observed on a target population,

$$
\begin{aligned}
& f\left(x_{s} y_{s}, x_{p}, y_{p} \mid s=p\right) \\
& \approx\left\{\frac{1}{4 \pi c^{2} \overline{x y} \sqrt{1-\rho^{2}}} e-\frac{1}{4 c^{2}\left(1-\rho^{2}\right)}\left[\frac{\left(x_{s}-x_{p}\right)^{2}}{\bar{x}^{2}}-\frac{2 \rho\left(x_{s}-x_{p}\right)\left(y_{s}-y_{p}\right)}{\overline{x y}}+\frac{\left(y_{s}-y_{p}\right)^{2}}{\bar{y}^{2}}\right]\right\} \\
& \times\left\{h(\bar{x}, \bar{y})\left[1-c^{2}\left(1+\frac{1}{2} \rho\right)\right]-c^{2}\left[\frac{\partial h(\bar{x}, \bar{y})}{\partial \bar{x}} \bar{x}+\frac{\partial h(\bar{x}, \bar{y})}{\partial \bar{y}} \bar{y}\right.\right. \\
& \left.\left.+\frac{\partial^{2} h(\bar{x}, \bar{y})}{\partial \bar{x}^{2}} \frac{\bar{x}^{2}}{4}+\frac{\partial^{2} h(\bar{x}, \bar{y})}{\partial \bar{y}^{2}} \frac{\bar{y}^{2}}{4}+\frac{\partial^{2} h(\bar{x}, \bar{y})}{\partial \bar{x} \partial \bar{y}} \frac{\overline{x y}}{2} \rho\right]\right\},
\end{aligned}
$$

where $\bar{x}=\left(x_{s}+x_{p}\right) / 2$ and $\bar{y}=\left(y_{s}+y_{p}\right) / 2$ serve as approximations for $t$ and $u, c^{4}$ terms have been eliminated and $\rho$ is a function of $\bar{x}-\bar{y}$. The first term in braces for Eq 4 indicates what one would expect: the likelihood that the true fragment lengths are identical $(s=p)$ depends on the size of the discrepancy between the matched pairs of fragment lengths. In other words, as the values of $\left(x_{s}-x_{p}\right)^{2}$ and $\left(y_{s}-y_{p}\right)^{2}$ increase, the likelihood decreases. For a given $t, X_{s}-X_{p}$ is distributed normally with a mean of zero and $2 c^{2} t^{2}$ is the variance.

With $\rho$ positive, as the research has shown, differences of the same sign for both alleles $\left(x_{s}-x_{p}\right.$ and $y_{s}-y_{p}$ are both positive or both negative) yield larger values for the likelihood of the identity model, than when the differences are of opposite sign. Therefore, both the size of the two differences and their pattern affects the likelihood of the identity model.

When $x_{s}=y_{s}$ and $x_{p}=y_{p}$ (both samples appear homozygous), the bivariate normal density can be replaced by the univariate normal density of $x_{s}-x_{p}$ which has mean zero and approximate variance $2 c^{2} \bar{x}^{2}[9,12]$. Berry et al. [8] consider other more complex problems with single banded profiles.

The error of the approximation in Eq 4 was under $1 \%$ across a wide range of values 
for a bivariate normal $g(t, u)$. The likelihood ratio equations of Berry et al. [8] and Devlin et al. [9] differ from our approximation in the way the bivariate density of true fragment lengths $g(t, u)$ is estimated. Their equations are similar to ours because all three assume bivariate normal measurement error. We smooth the bivariate density of the observables $[h(x, y)]$ first and then indirectly correct for the natural smoothing that occurs because of measurement error. This avoids the criticism Devlin et al. [9] made against the smoothing technique of Berry et al. [8], which may lead to oversmoothing spikes in the density of true fragment lengths. Evett et al. [10-12] provided an approximation equation similar to ours that does not consider the integration in Eq 3. As a result their equation does not include the correction factors in the second term in braces. We have, however, found our corrections to be mainly small and so our equations provide some justification for their simpler approximation. Evett et al. [12] also provide a detailed empirical analysis of error variance and correlation. The value of $\mathrm{Eq} 4$ is, therefore, its avoidance of the numerical integration in Berry et al. [8] and Devlin et al. [9] by an accurate approximation. Both our equations and those of Berry et al. [8] allow for dependence between alleles.

\section{The Likelihood for the Non-identity Model}

Under this model, true fragment lengths for the suspect and crime-scene samples are independent $\left(T_{s}, U_{s}\right.$ independent of $\left.T_{p}, U_{p}\right)$, and the density of the four observations can be expressed as:

$$
\begin{aligned}
f\left(x_{s}, x_{p}, y_{s}, y_{p} \mid s \neq p\right) & =\iint g\left(t_{s}, u_{s}\right) \phi\left(x_{s}, y_{s} \mid t_{s}, u_{s}\right) d t_{s} d u_{s} \\
& \iint g\left(t_{p}, u_{p}\right) \phi\left(x_{p}, y_{p} \mid t_{p}, u_{p}\right) d t_{p} d u_{p} \\
& =h\left(x_{s}, y_{s}\right) \cdot h\left(x_{p}, y_{p}\right),
\end{aligned}
$$

where the simple form involving the product of the two observable bivariate densities, one for the suspect sample and one for the crime sample, follows from Eq 1 and the independence assumption. Measurement variation across gels contributes to observed variation across a population's fragment lengths. If this is substantial, a correction in $h(x, y)$ should be used.

The controversy over the "correct" sub-population $[1,4-6]$ for determining chancematching probabilities is relevant to the estimation of $h(\bar{x}, \bar{y})$ in Eq 4 and $h\left(x_{s}, y_{s}\right)$ and $h\left(x_{p}, y_{p}\right)$ in Eq 5. For the common situation in which the sample at the crime scene is compared to the suspect's DNA, an estimate of $h(\bar{x}, \bar{y})$ in Eq 4 (numerator of the LR) and $h\left(x_{s}, y_{s}\right)$ in Eq 5 (denominator of the LR) could be based on any target population with which the suspect might be identified. This is because the numerator model assumes identity of the suspect's DNA and that found at the crime scene. An estimate of $h\left(x_{p}, y_{p}\right)$ in Eq 5 could be based on any target population with which the perpetrator might be identified regardless of the suspect, because the model for this equation assumes independence of the suspect and perpetrator. A sensitivity analysis that calculates LRs based on various target populations seems indispensable. For the situation in which the crime sample is of the victim's DNA and is to be matched with stains found on the suspect, similar reasoning would be used.

\section{Likelihood Ratio for the Full Profle of Fragment Lengths}

From the Lifecodes' data made available to us, correlations of fragment lengths for the three probes (on three different chromosomes) across three races were all near zero. 
From these correlations and past research [2,3], it seemed appropriate to assume that true fragment lengths across the three probes are independent. Although our LR does not require it, we also assumed independence of errors across probes for our calculations. (See Evett et al. [12] who use across probe error correlations in their calculations.) Given these assumptions, the product of the likelihood ratios obtained from each probe is the likelihood ratio for the three probes taken together.

\section{Likelihood Ratios for Selected Profiles}

The Lifecodes' data made available to us consisted of short and long fragment length measurements for the three loci D2S44, D17S79, and D14S13. The bivariate densities, $h(x, y)$, for the three loci were smoothed using a kernel-type method referred to as average shifted histograms [23]. Figure 1 is the estimated density for D2S44 and is based on a Caucasian data set $(n=1529)$. Using such smoothed estimates for the $h(x, y)$, we calculated likelihood ratios for selected profiles in order to reveal their sensitivity to the size and pattern of discrepancies between profiles.

Table 1 provides likelihood ratios for four different degrees of discrepancy between the two fragment length profiles. Observations were chosen so that the bivariate densities of the average of the fragment lengths for each probe were mid-way between the highest density and zero in the Caucasian data set. The average value of the two alleles for D2S44 were 10.82 and 12.67 kilobases, for D17S79 they were 3.39 and 3.70 , and for D14S13 they were 5.22 and 5.64. The first row of Table 1, labeled with a zero, is for the case when there is no discrepancy between the observed fragment lengths from the two samples $\left(x_{s}=x_{p}, y_{s}=y_{p}\right.$ for each of the three probes). With zero discrepancy, the value of the likelihood ratio for D2S44 is 99 , for D17S79 it is 48 , and for D14S13 it is 236. The product of these indicates that when the suspect and crime samples' fragment length measurements are the same across the three probes, the likelihood for the identity

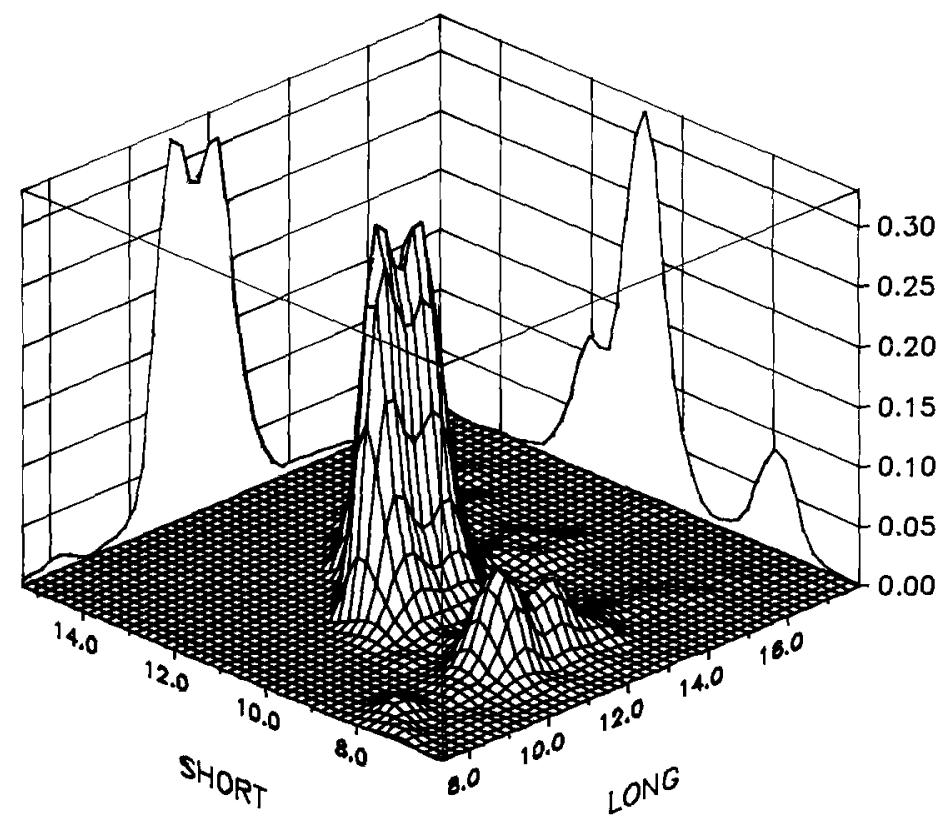

FIG. 1-Smoothed Bivariate Density, h(x,y), for D2S44 based on 1529 Caucasians. 
TABLE 1-Likelihood ratios as a function of four discrepancy sizes for each of three probes with $\mathrm{\rho}=0^{a}$

\begin{tabular}{ccccc}
\hline $\begin{array}{l}\text { \# of Std. Error Diffs. } \\
\text { between } x_{s} \text { and } x_{p} \text { and } \\
y_{s} \text { and } y_{p}\end{array}$ & $\begin{array}{c}\text { P2S44 } \\
\text { (density = 0.17) }\end{array}$ & $\begin{array}{c}\text { D17S79 } \\
\text { (density = 3.7) }\end{array}$ & $\begin{array}{c}\text { D14S13 } \\
\text { (density = 0.32) }\end{array}$ & $\begin{array}{c}\text { Product } \\
\text { of LRs }\end{array}$ \\
\hline 0 & 99. & 48. & 236. & $1.1 \times 10^{6}$ \\
1 & 62. & 29. & 142. & $2.6 \times 10^{5}$ \\
2 & 16. & 9.2 & 33. & 4858. \\
3 & 1.3 & .45 & 2.7 & 1.6 \\
4 & .04 & .02 & .08 & .00006 \\
\hline
\end{tabular}

$\bar{a}, \bar{y}$ observations were chosen for each probe which had a bivariate density $1 / 2$ as large as the highest density.

model is one-million times greater than the likelihood for the non-identity model. The next two rows provide LRs for discrepancies of one and two standard errors of measurement, respectively. Note that the LRs for each probe in these two rows are considerably reduced, but their products across the probes are still large. When the difference between the observed fragment lengths of the suspect and the crime samples are three or four standard errors of measurement, the evidence is inconclusive at best.

The LRs in Table 1 were calculated with $\rho=0$. They are provided to isolate the effect of the size of the discrepancies from the pattern of the discrepancies. When $\rho=0$, differences of the same or opposite sign for the two alleles yield essentially the same LR. Table 2 provides LRs with $\rho$ fixed to .9 to reveal the effect of the high error correlations that have been reported $[7-9,12]$. The same means $(\bar{x}, \bar{y})$ were used as those used for Table 1. Table 2 makes a distinction between two possible patterns. For example, the first row of the table provides the LRs when $x_{s}-x_{p}$ and $y_{s}-y_{p}$ are both positive and equal to one standard error of measurement (labeled $\mathrm{C}$ for consistent pattern). Note that the LRs in this row are higher than the corresponding LRs in Table 1 for a one standard error difference. This is true because deviations of the same size and sign (band shift) are more likely with highly correlated measurement error $(\rho=0.9)$ than with $\rho=0$. The effect of the opposite (inconsistent) pattern is apparent in the next row. In this row, the difference between $x_{s}$ and $x_{p}$ is -1 standard error and the difference between $y_{s}$ and $y_{p}$ is 1 standard error. The much lower LRs in the second row are

TABLE 2-Likelihood ratios with $\rho=0.9$ as a function of two patterns and four sizes of discrepancies.

\begin{tabular}{|c|c|c|c|c|c|}
\hline \multirow[b]{2}{*}{ Pattern } & \multicolumn{2}{|c|}{ Size in Std. Err. } & \multicolumn{3}{|c|}{ Probe } \\
\hline & $x_{s}-x_{p}$ & $y_{s}-y_{p}$ & D2S44 & D17S79 & D14S13 \\
\hline $\mathbf{C}^{a}$ & 1 & 1 & 180 & 83 & 415 \\
\hline $\mathbf{I}^{a}$ & -1 & 1 & 1.7 & 0.82 & 3.2 \\
\hline C & 2 & 2 & 87 & 34 & 192 \\
\hline$I$ & -2 & 2 & $2 \times 10^{-6}$ & $2 \times 10^{-4}$ & $1 \times 10^{-6}$ \\
\hline C & 3 & 3 & 23 & 9.3 & 51 \\
\hline $\mathrm{I}$ & -3 & 3 & $9 \times 10^{-17}$ & $1 \times 10^{-17}$ & $1 \times 10^{-17}$ \\
\hline $\mathrm{C}$ & 4 & 4 & 3.9 & 1.6 & 8.2 \\
\hline $\mathrm{I}$ & -4 & 4 & $4 \times 10^{-32}$ & $2 \times 10^{-32}$ & $8 \times 10^{-33}$ \\
\hline I & -0.5 & 0.5 & 67 & 33 & 154 \\
\hline
\end{tabular}

${ }^{a} \mathrm{C}$ is a pattern that is consistent with the positive correlation of errors; $\mathrm{I}$ is a pattern that is inconsistent. 
explained by a small likelihood of observing differences of opposite sign when $\rho=0.9$. In other words, these differences are inconsistent with the phenomena of band shifting and so is inconsistent with the identity model. The difference between the LRs for the 2,3 , and 4 standard error differences are much more pronounced and reveal the critical role played by the pattern of differences. The last row in Table 2 shows that when the difference is quite small ( 0.5 standard errors) but inconsistent, relatively large LR values are still obtained. Note, however, that these LR values are similar to the ones in row three for the two-standard-error discrepancies with a consistent pattern.

For the profiles in Tables 1 and 2, chance-matching probabilities were provided to us by Lifecodes Corporation. They were based on the three-standard-error statistical-matching criteria and are as follows: For the D2S44 probe the chance-matching probability for both alleles based on the Caucasian population is 0.04 , for D17S79 it is 0.04 , and for D14S13 it is .018 , yielding an overall chance-matching probability of $3 \times 10^{-5}$. For a three standard error difference between profiles, the product of the LRs in Table 1 was 1.6 implying such evidence is inconclusive. For all the opposite sign patterns in Table 2 , the LRs indicate the evidence is inconclusive or strongly against identity. We expect that experts would make decisions consistent with the values of the LRs, that is, profiles that differ by three or four standard errors for every fragment length $(1.8 \%$ to $2.4 \%$ of the fragment lengths) and that do not show a consistent band-shifting pattern would be considered inconclusive or against identity by an expert. Thus, a statistical-matching criteria of three- or four-standard errors breaks down when applied in a sterile manner. A dilemma is created by the match-binning approach, which is that chance-matching probabilities that are calculated by assuming strict adherence to the statistical matching criteria, but experts can not rely on these criteria as made obvious in our tables by the wide range of LRs from profiles that meet these criteria. The low values of the LR for three- and four-standard-error differences is a warning against using more liberal (inclusive) statistical-matching criteria.

It is difficult to design a truly generalizable study that compares the accuracy of LR and match-binning approaches. Studies based on a comparison of statistical matching (rather than expert matching) to likelihood ratios have shown that likelihood ratios are more accurate in correctly classifying identical and non-identical profiles. This is as expected, because the information in the sizes and pattern of discrepancies is utilized by the likelihood ratio approach, but ignored by a sterile statistical-matching approach.

\section{Estimation Error}

The likelihood ratios are subject to sampling error. It is appropriate to provide a range of possible values as a function of sampling error in the estimation of $h(x, y)$ as well as the measurement error parameters. At the least, the lower limit of such an interval should be made known. For such a complex statistic like the $L R$, intervals based on the bootstrap procedure could be used [24]. Results in Devlin et al. indicate that their LRs are relatively accurate for a population sample size of just 125 [9].

The value for the measurement error variance that was used here $(.6 \%)$ is based on Lifecodes methods and uncontaminated samples. Such an estimate may be specific to their studies, and it seems clear that each laboratory needs to determine its own error variance on a continuing basis. The influence of the correlation of error $(p)$ on determining the likelihood of distinct patterns of discrepancies, and the possibility of bias and non-normal errors (because of degradation of samples) suggest that a continuing investigation of the distribution of error is needed and that the use of the likelihood ratio without expert judgement is to be avoided. Thus far, research that has tested the effects of various conditions on DNA samples has shown that readable samples are consistent with untreated controls and that the error variance is not increased [17-19]. 


\section{Conclusion}

The likelihood ratio approach does not require an initial decision about whether or not two profiles match. Instead, it quantifies the strength of the evidence in favor of identity by its sensitivity to the sizes and pattern of discrepancies between profiles. The wide range of likelihood ratios reported in Tables 1 and 2 suggest that this sensitivity is most critical when the two profiles approach the limits of the published statistical matching criteria.

The theoretical foundation of likelihood ratios is somewhat complex for jurors, but their numerical values can be labelled with broad descriptions such as "very strong evidence of identity." Descriptions should be followed by reports on the proportion of times such strong results have been obtained from two different persons in large databases. The effects on the value of the likelihood ratio of choices for target population and assumptions about the measurement error distribution should also be provided.

\section{Acknowledgment}

Early work was supported by Gennan Corporation, Akron, OH. We thank C. Kandiko for his contributions during this time. We also thank I. Balazs of Lifecodes Corporation, Valhalla, NY, who provided the data sets and comments on the paper and M. Baird also of Lifecodes who provided chance-matching probabilities for the example profiles. Suggested revisions by anonymous reviewers were also appreciated. All errors are those of the authors.

\section{References}

[1] Risch, N. J. and Devlin, B., "On the Probability of Matching DNA Fingerprints," Science, Vol. 255, Feb. 1992, pp. 717-720.

[2] Budowle, B., Giusti, A. M., Waye, J. S., Baechtel, F. S., Fourney, R. M., Adams, D. E., Presley, L. A., Deadman, H. A., and Monson, K. L., "Fixed-Bin Analysis for Statistical Evaluation of Continuous Distributions of Allelic Data from VNTR Loci, for Use in Forensic Comparisons," American Joumal of Human Genetics, Vol. 48, 1991, pp. 841-855.

[3] Balazs, I., Baird, M., Clyne, M., and Meade, E., "Human Population Genetics Studies of Five Hypervariable DNA Loci," American Journal of Human Genetics, Vol. 44, 1989, pp. $182-190$.

[4] Chakraborty, R. and Kidd, K. K., "The Utility of DNA Typing in Forensic Work," Science, Vol. 254, Dec. 1991, pp. 1735-1739.

[5] Lewontin, R. C. and Hartl, D. C., "Population Genetics in Forensic DNA Typing," Science, Vol. 254, Dec. 1991, pp. 1745-1750.

[6] National Academy of Sciences Committee on, DNA Technology in Forensic Science, National Academy Press, Washington, D.C., 1992.

[7] Berry, D. A., "Inferences Using DNA Profiling in Forensic Identification and Paternity Cases," Statistical Science, Vol. 6, 1991, pp. 175-189.

[8] Berry, D. A., Evett, I., and Pinchin, R., "Statistical Inference in Crime Investigations Using Deoxyribonucleic Acid Profiling," Journal of the Royal Statistical Society (Series C), Vol. 41, No. 3, pp. 499-531.

[9] Devlin, B., Risch, N., and Roeder, K., “Forensic Inference From DNA Fingerprints," Journal of the American Statistical Association, Vol. 87, No. 418, June 1992, pp. 337-350.

[10] Evett, I., Werett, D., Pinchin, R., and Gill, P., "Bayesian Analysis of Single Locus DNA Profiles," Proceedings of the International Symposium on Human Identification 1989, Promega Corp., Madison, Wisconsin, 1990.

[11] Buckleton, J., Walsh, K., and Triggs, C., "A Continuous Model for Interpreting the Positions of Bands in DNA Locus-Specific Work," Journal of the Forensic Science Society, Vol. 31, No. 3, 1991, pp. 353-363.

[12] Evett, I., Svanage, J. K., and Pinchin, R., "An Efficient Statistical Procedure for Interpreting DNA Single Locus Profiling Data in. Crime Cases," Journal of the Forensic Science Society, Vol. 32, No. 4, 1992, pp. 307-326. 
[13] Lindley, D. V., "A Problem in Forensic Science," Biometrika, Vol. 64, No. 2, 1977, pp. 207213.

[14] Morris, J. W., Sanda, A. I., and Glassberg, J., "Biostatistical Evaluation of Evidence from. Continuous Allele Frequency Distribution Deoxyribonucleic Acid (DNA) Probes in Reference to Disputed Paternity and Identity,' Journal of Forensic Sciences, Vol. 34, No. 6, Nov. 1989, pp. 1311-1317.

[15] Gjertson, D. W., Mickey, M. R., Hopfield, J., Takenouchi, T., and Terasaki, P. I., 'Calculation of Probability of Paternity Using DNA Sequences," American Joumal of Human Genetics, Vol. 43, 1988, pp. 860-869.

[16] Baird, M., Balazs, I., Giusti, A., Miyazeki, L, Nicholas, L., Wexler, K., Kanter, E, Glassberg, J., Allen, F., Rubenstein, P., and Sussman, L., "Allele Frequency Distribution of Two Highly Polymorphic DNA Sequences in Three Ethnic Groups and Its Application to the Determination of Paternity," American Journal of Human Genetics, Vol. 39, 1986, pp. 207-213.

[17] McNally, M. S., Shaler, R. C., Baird, M., Balazs, I., DeForest, P., and Kobilinsky, L., "Evaluation of Deoxyribonucleic Acid (DNA) Isolated from Human Bloodstains Exposed to Ultraviolet Light, Heat, Humidity, and Soil Contamination," Journal of Forensic Sciences, Vol. 34, No. 5, Sept. 1989 , pp. 1059-1069.

[18] McNally, M. S., Shaler, R. C., Baird, M., Balazs, I., Kobilinsky, L., and DeForest, P., "The Effects of Environment and Substrata on Deoxyribonucleic Acid (DNA): The Use of Casework Samples from New York City,' Journal of Forensic Sciences, Vol. 34, No. 5, Sept. 1989, pp. 1070-1077.

[19] Adams, D. E., Presley, L. A., Baumstark, A. L., Hensley, K. W., Hill, A. L., Anoe, K. S., Campbell, P. A., McLaughlin, C. M., Budowle, B., Giusti, A. M., Smerick, J. M., and Bachtel, F. S., "Deoxyribonucleic Acid (DNA) Analysis by Restriction Fragment Length Polymorphisms of Blood and Other Body Fluid Stains Subjected to Contamination and Environmental Insults," Journal of Forensic Sciences, Vol. 36, No. 5, Sept. 1991, pp. 1284-1298.

[20] Technical Comments on Forensic DNA Tests and Hardy-Weinberg Equilibrium, Science, Vol. 253, 1991, p. 1037.

[21] Trumpler, R. J. and Weaver, H. F., Statistical Astronomy, Univ. of California Press, Berkeley, 1953.

[22] Lord, F. M. and Novick, M. R., Statistical Theories of Mental Test Scores, Addison-Wesley, Reading, Mass, 1968.

[23] Scott, D. W., “Average Shifted Histograms: Effective Nonparametric Density Estimators in Several Dimensions,' Annals of Statistics, Vol. 13, No. 3, 1985, pp. 1024-1040.

[24] Efron, B. and Tibshirani, R., "Bootstrap Methods for Standard Errors, Confidence Intervals and Other Measures of Statistical Accuracy,' Statistical Science, Vol. 1, No. 1, 1986, pp. 5477.

Address requests for reprints or additional information to

David Jarjoura, Ph.D.

Northeastern Ohio Universities College of Medicine

P.O. Box 95

Rootstown, OH 44272 